\title{
TITLE:
}

\section{Chronobiology of micturition: putative role of the circadian clock.}

$\operatorname{AUTHOR}(\mathrm{S})$ :

Negoro, Hiromitsu; Kanematsu, Akihiro; Yoshimura, Koji; Ogawa, Osamu

\section{CITATION:}

Negoro, Hiromitsu ... [et al]. Chronobiology of micturition: putative role of the circadian clock.. The Journal of urology 2013, 190(3): 843-849

ISSUE DATE:

2013-09

URL:

http://hdl.handle.net/2433/178676

\section{RIGHT:}

(c) 2013 American Urological Association Education and Research, Inc. Published by Elsevier Inc.; This is not the published version. Please cite only the published version.; こ の論文は出版社版でありません。引用の際には出版社版をご確認ご利用ください。 


\section{Chronobiology of micturition: putative role of the circadian clock}

Hiromitsu Negoro, ${ }^{1}$ Akihiro Kanematsu, ${ }^{1,2}$ Koji Yoshimura ${ }^{1}$ and Osamu Ogawa ${ }^{1} *$

${ }^{1}$ Department of Urology, Graduate School of Medicine, Kyoto University, Sakyo, Kyoto 606-8507, Japan;

${ }^{2}$ Department of Urology, Hyogo College of Medicine, Nishinomiya, Hyogo 663-8501, Japan

*Correspondence should be addressed to O.O. (ogawao@kuhp.kyoto-u.ac.jp).

Running head: Chronobiology and micturition rhythm

Key words: bladder, micturition, rhythm, diurnal, circadian 


\section{Abstract}

Purpose: Mammals urinate less frequently during sleep period than during awake period, which is modulated by triad factors; decreased arousal level in the brain, decreased urine production rate from the kidneys, and increased functional bladder capacity during sleep. The circadian clock ('the clock') is genetic transcription-translation feedback machinery. The clock exists in most organs and cells, termed 'peripheral clock', which is orchestrated by the central clock in the suprachiasmatic nucleus of the brain. In this review we discuss the linkage between the day-and-night change in micturition frequency and the genetic rhythm maintained by the clock system, focusing on brain, kidney and bladder.

Material and Methods: We performed inclusive review of the literature on diurnal change in micturition frequency, urine volume, functional bladder capacity and urodynamics in humans and rodents, and related it with recent basic biological findings about the circadian clock.

Results: In humans, various behavioral studies demonstrated diurnal functional change in kidney and bladder, and conversely, patients with nocturnal enuresis and nocturia show impairment of these triad factors. Rats and mice, which are 
nocturnal animals, also show rhythm in micturition frequency, decreased during daytime, which is sleep phase for them. Mice having genetically defective circadian clock system show impaired physiological rhythms in the triad factors. Existence of the circadian clock has been proven in brain, kidney and also in the bladder, in which exist thousands of circadian oscillating genes. In the kidney, they include genes involved in regulation of water and major electrolytes and in the bladder, a gene associated with regulation of bladder capacity, connexin 43 .

Conclusion: Recent progress in molecular biology about the circadian clock provides an opportunity for investigating genetic basis of micturition rhythm, or impairment of the rhythm in nocturnal enuresis and nocturia. If this approach is to be translated clinically, one strategy is to analyze and treat the triad of micturition factors as separate parts of one problem. The other way could be, if possible, to cope with these triad problems simultaneously by treating the circadian physiological rhythm itself. Discoveries reviewed here point toward further investigation of micturition rhythm by basic and translational chronobiology. 


\section{Introduction}

The day-night change in micturition frequency is essential for having a sound sleep for healthy humans. Studies on normal and abnormal micturition suggest that nocturnal micturition is prevented not only by sleep itself, but also by a decrease in urine production rate from the kidneys, and an increase in storage capacity of the urinary bladder (Figure 1). ${ }^{1-3}$ Disturbance of such coordination typically occurs in nocturnal enuresis in children or nocturia in elderly people. ${ }^{3}$ Nocturnal enuresis is seen in approximately $10 \%$ of 7 -year-old children with a spontaneous cure rate of $15 \%$ a year, and has a significant effect on patients' self-esteem. ${ }^{3,4}$ Nocturia is present in $60-90 \%$ of elderly people older than 60 years old, and significantly deteriorates their quality of life. ${ }^{5}$ However, the treatment of these patients in clinics is often limited to palliation because the precise mechanism underlying enuresis and nocturia is unclear.

Chronobiology is an emerging new approach for investigating disorders related to biological rhythm. The discovery of the circadian clock, which is genetic machinery generating a circadian rhythm in systemic organs, was a revolutionary finding that enabled researchers to analyze circadian biological phenomena from the standpoint of genetics. There is increasing evidence that behavior, physiology and metabolism in mammals are under control of the circadian clock. ${ }^{6-8}$ However, molecular 
chronobiological analysis of micturition has not been reported until recently. ${ }^{9,10}$

This Review article describes how the notion of the circadian clock and chronobiology sheds new light on day-night changes in micturition frequency in mammals, interpreted as the circadian biological rhythm. This is a novel paradigm for investigating the pathophysiology of nocturnal enuresis as immaturity in development of the rhythm, and nocturia as loss of the rhythm.

\section{Diurnal micturition rhythm}

\section{Humans}

Urination in neonates involves reflex voiding, which gradually becomes regulated by upper central neurons with postnatal development, accompanied by enlargement of bladder capacity. Control of day and nighttime micturition is established by school age, but at least $10 \%$ of school children have nocturnal enuresis. ${ }^{3}$ Studies have also shown that established diurnal micturition is gradually disturbed in many elderly people, which is known as nocturia. ${ }^{1,2}$ Although the pathogeneses of these two conditions have been separately discussed, enuresis and nocturia share a similar triad of features: an impaired day-night rhythm of arousal level, urine production, and bladder storage.

Impaired arousal level during sleep is observed in enuresis and nocturia. 
Studies with polysomnography have demonstrated that enuretic children are deep sleepers just at the time of urine loss, but are light sleepers during the rest of the sleep period. ${ }^{11,12}$ Impaired sleep has also been documented in elderly people with nocturia. ${ }^{5}$

Nocturnal polyuria is another common feature of enuresis and nocturia. Day-night variation in urine production is not simply caused by a change in oral intake of food and liquid, but is maintained in subjects under a constant routine, when food and drink are taken equally during 24 hours in constant dim light. ${ }^{13}$ However, kidneys of many enuretic children produce more urine when asleep than those of healthy children, resulting in use of anti-diuretic hormone as standard treatment for such types of cases. ${ }^{3}$ Abnormal diurnal rhythms of plasma vasopressin have also been reported in patients with enuresis. ${ }^{14}$ Similarly, elderly people with refractory nocturia often show excessive urine production and natriuresis during sleep. ${ }^{15}$

The same type of observation as impaired arousal level and urine production rate during sleep in enuresis and nocturia is documented for day-night change in functional bladder capacity. Normal healthy adults have a greater volume voided per micturition during midnight to morning (Figure 2, left), ${ }^{1,16,17}$ in parallel with a decrease in maximal flow rate in uroflowmetry. In contrast, less voided volume and a higher flow rate, representing greater detrusor tonus, are observed during afternoon to 
evening. ${ }^{16}$ These day-night changes in functional bladder capacity and maximal flow rate are interpreted as a "shift" of urinary bladder function between storage and elimination of urine within a day. Enuretic and healthy children have similar daytime holding-exercise volumes, but early-morning voiding volume in enuretic children is smaller than that in healthy children. ${ }^{18}$ In only $28 \%$ of enuretic children, early-morning voided volume reaches maximal volume voided per micturition in a day, as opposed to $72 \%$ of healthy children. Such reduction in nocturnal functional bladder capacity is an important feature of refractory nocturnal enuresis. ${ }^{3}$ This fact indicates that pathogenesis of enuresis may not be related to the structural bladder size itself, but to the regulatory mechanism of diurnal change in functional bladder capacity instead. In parallel with this suggestion, elderly people have a significantly decreased capacity for normal desire to void during night compared with middle-aged men, ${ }^{17}$ and such a decrease in nighttime functional bladder capacity is also a hallmark of nocturia.

\section{Rodents as models}

Rodents are nocturnal animals, and therefore, the sleep-awake cycle is opposite from humans in the light-dark cycles. Early studies in rodents on the micturition cycle were performed using rats, mainly for technical reasons compared with mice. Studies showed 
that the bladder capacity of rats is increased in the sleep phase and decreased in the active phase, as demonstrated by an electric balance system under free-moving conditions, ${ }^{10,19}$ or by a cystometrogram without being affected by the urine production rate (Figure 2, middle). ${ }^{20}$

For studying genetic mechanisms of micturition, genetically-modified mice are a useful tool, but their bladder capacity is so minute that researchers employed a urine stain on filter paper as a surrogate of voided volume. ${ }^{21,22}$ This principle was further developed for enabling diachronic measurement and was named automated voided stain on paper (aVSOP) method. ${ }^{10,23}$ Micturition rhythm emerges with a urine production rhythm appearing at 3 to 4 weeks old, followed by maturation of a diurnal change in bladder capacity occurring around 5 weeks old..$^{23}$ This micturition rhythm, observed as that of humans and mice, is maintained in a constant dark condition (Figure 2, right), which indicates that mice have an internal control mechanism of micturition. Thus mice, young to adult, have become a model animal for studying micturition cycle.

\section{The circadian clock system}

The central master clock

Circadian rhythms of systemic activity are sustained even in the absence of 
environmental cues, and are often associated with an internal biological clock, i.e., the circadian clock. The master circadian clock is located in the suprachiasmatic nucleus (SCN) of the hypothalamus, and generates central circadian rhythm of the body. This region has a self-sustained circadian expression of clock genes, and also synchronizes the master clock to the environment, by receiving a light signal cycle from the retina. ${ }^{6,8}$

\section{Transcriptional circuits}

The molecular mechanism of the circadian clock in mammals was revealed with the discovery of the mouse Clock in 1994 and the human and mouse Period in 1997. At the molecular level, circadian clock oscillations are driven by transcription-translation feedback loops consisting of PER and CRY as negative components, and CLOCK and BMAL1 as positive components (Figure 3A). Rhythmic oscillations of these core loops are followed by the clock-associated oscillations of $D b p$ and Rev-erb $\alpha$, whose products tune the core loops and also control oscillations of many genes through D-box and RORE sites, respectively (Figure 3A). ${ }^{6-8}$

\section{Peripheral clocks}

Notably, this molecular clock system is present not only in the SCN, but also in most 
peripheral tissues and organs, such as the brain, lungs, heart, liver, pancreas, gastrointestinal tract, adrenal glands, skeletal muscles, kidneys, and others. ${ }^{24}$ Though the detailed interplay between SCN and peripheral clocks is not fully understood, peripheral clocks are tuned by signals from the SCN by three ways: 1) as neural signals via sympathetic/parasympathetic nerves; 2) as hormonal signals via the pineal body and adrenal glands; and 3) as indirect behavioral controls via feeding and sleep-awake cycles (Figure 3B). ${ }^{8,24}$

\section{The clock and diseases}

Emerging evidence indicates that disturbance of the circadian rhythm is linked to various pathological conditions in clinical and basic studies. For example, on the clinical side, polymorphisms of clock genes are associated with early or late chronotype, metabolic syndrome and cancer morbidity. ${ }^{25,26}$ Epidemiological studies suggest that shift workers have a higher incidence of diabetes, obesity, cardiovascular diseases, psychological disorders, gastrointestinal dysfunction and cancer. ${ }^{26,27}$ Basic studies have shown that dysfunction of the circadian clock is involved in salt-sensitive hypertension by dysregulated adrenal Hsd $3 \mathrm{~b} 6,{ }^{8}$ and in diabetes mellitus by impaired insulin secretion from $\beta$-cells. ${ }^{7}$ 


\section{The clock and micturition}

As noted above, the frequency of micturition is deeply associated with three factors: arousal level in the brain, urine production rate in the kidneys, and functional capacity in the bladder (Figure 1). A relation between the circadian clock with two of the three factors (arousal level and urine production rate) has been reported relatively earlier.

With regard to arousal level of brain during sleep, clock gene mutant mice have several sleep abnormalities. ${ }^{25}$ Clock mutant mice have reduced non-rapid eye movement (NREM) sleep and reduced total sleep time. Bmall knockout mice have increased sleep fragmentation, but they have longer REM and NREM periods, while Per1/Per2 double mutant mice show decreased REM and NREM sleep periods.

With regard to urine production in the kidneys, circadian excretion and reabsorption of water and major electrolytes in urine have been shown physiologically, ${ }^{28}$ while rise in plasma vasopressin at late sleep phase is modulated by the central circadian clock $^{14}$ and circadian oscillation in transcription of the key molecules for these steps in the kidneys has also been shown genetically. ${ }^{29,30}$ The examples of such genes are, $\mathrm{Na}^{+} / \mathrm{H}^{+}$exchanger, aquaporin-2, 3 and 4 , and type 2 vasopressin receptor that regulate water reabsorption, and many genes regulating 
electrolyte movement, such as epithelial sodium channel (ENaC)-mediated sodium transport, chloride movement across the epithelia, and potassium excretion. Clock knockout mice have a significant reduction in renal and urinary content of 20-hydroxyeicosatetraenoic acid, a powerful endogenous regulator of renal sodium reabsorption and potassium secretion, and they have a reduction in renal vascular tone. ${ }^{31}$ Perl regulates sodium transport genes, such as $\alpha E N a C$ and endothelin- $1,{ }^{32}$ and Perl and Per2 double knockout mice lose the diurnal rhythm of urine volume. ${ }^{9}$

\section{Circadian rhythm in the bladder}

\section{Discovery of the clock in the bladder}

The circadian clock regulation for the third component of the triad, the functional capacity in the bladder, has not been investigated until recently. ${ }^{10}$ The existence of a functional peripheral clock in the bladder was proven by three methods, as performed for other organs. The first method involved analyzing circadian expression of clock genes in the bladder in vivo, with and without modifications of clock genes. The urinary bladder has a strong oscillation rhythm in core clock genes, Per 2, Bmal1, Cryl/2 and Clock, and also in regulators, such as Rev-erb $\alpha / \beta$, Rorc, Dbp, Tef, Hlf, E4bp4 and Decl. The second method is the ex vivo culture system using Per $2^{\text {luciferase }}$ knock-in mice, 
carrying a PER2::LUC fusion protein, which was engineered to produce bioluminescence in the presence of luciferin when the clock gene Per2 is activated. This system provides strong evidence that the peripheral clock in the bladder functions without systemic cues. ${ }^{10,33,34}$ Notably, the luminescence of extracted bladder can be modulated by mechanical and/or chemical stimuli. ${ }^{33}$ The period of the circadian rhythm is different between organs as assessed by Per2 $2^{\text {luciferase }}$ mice as follows: in SCN it is $23.5 \mathrm{~h}$, in pancreas islets $23.6 \mathrm{~h}$, in the cornea $22.2 \mathrm{~h}$, in the kidney $24.8 \mathrm{~h}$, and in the urinary bladder it is $24.9 \mathrm{~h}$. In particular, this monitoring system has recently been applied for animals in vivo, in a noninvasive and diachronic manner. ${ }^{35}$ Use of this system is expected to reveal the detailed relation between the central/peripheral clock and the micturition rhythm, cross-linked to other genetic modified mice if possible. The third method is the serum-shock method for synchronizing the circadian rhythm of cultured cells in vitro. Primary cultured bladder smooth muscle cells show in vitro genetic oscillation for Per2, Bmal1, Rev-erbo, and others.

\section{Oscillating genes in the bladder}

Genes having circadian expression vary between tissues and organs. For example, expression levels of $8-10 \%$ of genes oscillate in a circadian manner in the heart and 
liver, but only $0.3 \%$ of genes commonly oscillate in both organs. ${ }^{36}$ Oscillatory genes are frequently associated with a crucial function in organs In the liver, oscillating genes encode key steps of metabolism as sterol regulatory element-binding protein (SREBPS), involved in the master pathway of hepatic lipid metabolism and Nocturnin, involved in lipogenesis and energy homeostasis. ${ }^{7,37}$ In the urinary bladder also, thousands of genes oscillate. ${ }^{10}$ When oscillatory genes in the urinary bladder are classified by the biological process in the third level of the Gene Ontology hierarchy, ${ }^{38}$ most oscillatory genes are involved in "circadian rhythm", "regulation of biological process", "cellular and nitrogen compound metabolic process" and "response to stimulus". The bladder functions in a dynamic circadian manner with these biological processes. Connexin 43, a gap-junction protein in the urinary bladder and associated with sensitivity of bladder muscle, ${ }^{39}$ is in both "regulation of biological process" and "response to stimulus" may have important role in circadian change in bladder function as reported. ${ }^{10}$

\section{Clinical translation}

The question can be asked: "Is the circadian clock system related to micturition disorder?" This should be the case, because Cry-null mice and Per1/Per2 double knockout mice, which have a dysfunctional circadian clock, completely lose diurnal 
rhythm of micturition, urine production and functional bladder capacity. ${ }^{10,34,39}$ These findings correspond to dysfunction in the diurnal micturition rhythm observed in patients with nocturnal enuresis and nocturia. Interestingly, a previous study reported a correlation between enuresis in children and nocturia in their mothers. ${ }^{40}$ A recent study also showed that nocturnal enuresis is carried over as adult LUTS. ${ }^{41}$ Studies from Denmark suggest a strong genetic linkage of enuresis with identification of chromosomal loci for responsible genes. ${ }^{4}$ Taken together, these findings indicate that disorders in the micturition cycle could be genetic events that encompass the whole life of an individual, which may be "masked" during early adulthood after adolescence. Such clinical findings suggest that applying a novel paradigm of chronobiology and the notion of the circadian clock system could be a promising approach to elucidate the genetic etiology of these diseases.

There are two strategies for treatment of enuresis and nocturia if the chronobiological approach is to be applied for these micturition rhythm disorders. One strategy is to analyze and treat each factor of this triad (the brain, kidney and bladder) as separate parts of one problem. For nocturic elderly people with waking early in the night and taking a time to return to sleep, using a rapid-acting hypnotic is a choice of treatment. For altering the urine production rhythm in the kidneys, desmopressin is 
available in market for enuresis and nocturia. Recipients of a denervated kidney in renal transplantation lose diurnal changes in urine production, which can be treated with daytime diuretics. $^{42}$ One could aim for inventing novel drugs to regain/suppress daytime/nighttime urine production based on circadian function of the kidney. For the bladder, one may investigate to develop a bladder-specific gap-junction inhibitor for treating patients with enuresis and nocturia. In addition, microarray data ${ }^{10}$ also present a list of oscillating bladder genes that could be candidate targets for inventing drugs aimed at nighttime bladder problems. Dealing with the triad separately or by a combination of multiple drugs may be a natural extension of the studies done thus far.

The other strategy for treatment of micturition rhythm disorders could be to cope with these triad problems simultaneously by treating the circadian rhythm itself if the circadian clock system governs the triad. Indeed, the triad is intricately intertwined with each other. ${ }^{5,43}$ If the major cause of enuresis and nocturia is dysfunction of the clock, targeting the clock itself could be a universal measure to treat these problems. If this is the case, it is important to identify a methodology or biomarker for measuring biological rhythm in each individual, ${ }^{44}$ corresponding to the micturition rhythm to evaluate whether dysfunction of the clock is the etiology of enuresis and nocturia. Development process of such micturition rhythm in normal human infant is another 
topic to be elucidated for further progress in this field, and we are currently working to devise a novel system for automatically recording infant micturition before toilet-training. It has been reported that nocturia is a significant risk factor for mortality, ${ }^{5}$ indicating that disturbance of the micturition rhythm may represent a disturbance of systemic homeostasis itself. Disruption of circadian rhythms seriously affects life-span, as shown in mice with mutant clock genes. ${ }^{45}$ Systemic diseases, such as diabetes, depression and hypertension, in which a link to the circadian clock has been documented, are often accompanied by each other and also with nocturia. ${ }^{5}$ Aging, the most definite risk factor of nocturia, is also strongly associated with the weakness of circadian clock function in the SCN and peripheral organs. ${ }^{45}$ Higher amplitude rhythms were restored and the life-span was extended when the fetal SCN was implanted in aged animals. ${ }^{45}$ Weakness of the circadian amplitude in behavior was observed in mice lacking the large conductance $\mathrm{Ca}^{2+}$-activated $\mathrm{K}^{+}$channel $(\mathrm{BK}),{ }^{46}$ whose expression was significantly decreased in smooth muscle during aging. ${ }^{47} \mathrm{BK}$ channel-null mice also showed overactive bladder and incontinence. ${ }^{21}$ In this scenario, appropriate control of the circadian rhythm may restore general health, including bladder function. Melatonin and ramelteon, its agonist, not only facilitate sleep but also entrain the circadian system, increasing bladder capacity and reducing bother of nocturia. ${ }^{48}$ An improvement in 
life-style significantly affects the symptoms of nocturia. ${ }^{49}$ Therefore, appropriate entrainments by bright light, feeding, exercise, melatonin agonists and upcoming novel medication acting on the products of clock-controlled genes or clock gene itself, could be new candidates for treatment and prevention of enuresis and nocturia.

In summary, studying micturition as a biological rhythm may not only improve the quality of life in children and elderly people, but may also uncover an unknown regulatory mechanism of the human body by the clock, which affects daily life. Currently, our research group is participating in a comprehensive and prospective population-based cohort study aimed at systemic health status, ${ }^{50}$ which includes a genetic and behavioral survey of LUTS. Such an approach may determine the implication of biological rhythm on LUTS and life in general.

\section{Conclusion}

The findings discussed in this Review article highlight a novel chronobiological approach to micturition rhythm. Continued basic and clinical researches are required to advance chronobiology of micturition, and this approach warrants further investigation for disorders characterized as disorders of micturition rhythm: nocturnal enuresis and nocturia. 


\section{Acknowledgments}

We thank H. Okamura, Y. Tabata, and T. Oura for valuable discussion. This research was supported by Grant-in-Aids for Scientific Research 23659756 and 24659719 from the Japan Society for the Promotion of Science.

\section{Author contributions}

H. Negoro researched the data for the article and wrote the manuscript. A. Kanematsu planned and supervised the study, and prepared the manuscript. K. Yoshimura and O. Ogawa contributed to the discussions of content. All authors reviewed and edited the manuscript before submission.

\section{Competing financial interests}

The authors declare no competing financial interests.

\section{REFERENCES}


1. Nakamura S, Kobayashi Y, Tozuka K et al: Circadian changes in urine volume and frequency in elderly men. J Urol 1996; 156: 1275-1279.

2. Bosch JL, Weiss JP: The prevalence and causes of nocturia. J Urol 2010; 184: 440-446.

3. Neveus T: Diagnosis and management of nocturnal enuresis. Curr Opin Pediatr 2009; 21: 199-202.

4. von Gontard A, Schaumburg H, Hollmann E et al: The genetics of enuresis: a review. J Urol 2001; 166: 2438-2443.

5. Yoshimura K: Correlates for nocturia: a review of epidemiological studies. Int $\mathbf{J}$ Urol 2012; 19: 317-329.

6. Dibner C, Schibler U, Albrecht U: The mammalian circadian timing system: organization and coordination of central and peripheral clocks. Annu Rev Physiol 2010; 72: 517-549.

7. Bass J, Takahashi JS: Circadian integration of metabolism and energetics. Science 2010; 330: 1349-1354.

8. Okamura H, Doi M, Fustin JM et al: Mammalian circadian clock system:

Molecular mechanisms for pharmaceutical and medical sciences. Adv Drug Deliv Rev 2010; 62: 876-884.

9. Noh JY, Han DH, Yoon JA et al: Circadian rhythms in urinary functions: possible roles of circadian clocks? Int Neurourol J 2011; 15: 64-73.

10. Negoro H, Kanematsu A, Doi M et al: Involvement of urinary bladder Connexin43 and the circadian clock in coordination of diurnal micturition rhythm. Nat Commun 2012; 3: 809.

11. Yeung CK, Diao M, Sreedhar B: Cortical arousal in children with severe enuresis. N Engl J Med 2008; 358: 2414-2415.

12. Neveus T, Stenberg A, Lackgren $G$ et al: Sleep of children with enuresis: a polysomnographic study. Pediatrics 1999; 103: 1193-1197.

13. Boivin DB, Duffy JF, Kronauer RE et al: Sensitivity of the human circadian pacemaker to moderately bright light. J Biol Rhythms 1994; 9: 315-331.

14. Trudel E, Bourque CW: Circadian modulation of osmoregulated firing in rat supraoptic nucleus neurones. J Neuroendocrinol 2012; 24: 577-586.

15. Matthiesen TB, Rittig S, Norgaard JP et al: Nocturnal polyuria and natriuresis in male patients with nocturia and lower urinary tract symptoms. J Urol 1996; 156: 1292-1299. 
16. Poulsen EU, Kirkeby HJ: Home-monitoring of uroflow in normal male adolescents. Relation between flow-curve, voided volume and time of day. Scand J Urol Nephrol Suppl 1988; 114: 58-62.

17. Satoh W, Nakada T: Characteristics of circadian change in urinary frequency, bladder capacity and residual urine volume in elderly men with lower urinary tract symptoms. Nurs Health Sci 1999; 1: 125-129.

18. Van Hoeck K, Bael A, Lax $\mathrm{H}$ et al: Urine output rate and maximum volume voided in school-age children with and without nocturnal enuresis. J Pediatr 2007; 151: 575-580.

19. Schmidt F, Yoshimura Y, Ni RX et al: Influence of gender on the diurnal variation of urine production and micturition characteristics of the rat. Neurourol Urodyn 2001; 20: 287-295.

20. Herrera GM, Meredith AL: Diurnal variation in urodynamics of rat. PLoS One 2010; 5: e12298.

21. Meredith AL, Thorneloe KS, Werner ME et al: Overactive bladder and incontinence in the absence of the BK large conductance $\mathrm{Ca} 2+$-activated $\mathrm{K}+$ channel. J Biol Chem 2004; 279: 36746-36752.

22. Sugino Y, Kanematsu A, Hayashi Y et al: Voided stain on paper method for analysis of mouse urination. Neurourol Urodyn 2008; 27: 548-552.

23. Negoro H, Kanematsu A, Matsuo M et al: Development of diurnal micturition pattern in mice after weaning. J Urol 2013; 189: 740-746.

24. Richards J, Gumz ML: Advances in understanding the peripheral circadian clocks. FASEB J 2012; 26: 3602-3613.

25. Landgraf D, Shostak A, Oster H: Clock genes and sleep. Pflugers Arch 2012; 463: 3-14.

26. Innominato PF, Levi FA, Bjarnason GA: Chronotherapy and the molecular clock: Clinical implications in oncology. Adv Drug Deliv Rev 2010; 62: 979-1001.

27. Wright KP Jr, Bogan RK, Wyatt JK: Shift work and the assessment and management of shift work disorder (SWD). Sleep Med Rev 2013; 17: 41-54.

28. Minors DS, Waterhouse JM: Circadian rhythms of urinary excretion: the relationship between the amount excreted and the circadian changes. J Physiol 1982; 327: 39-51.

29. Saifur Rohman M, Emoto N, Nonaka H et al: Circadian clock genes directly regulate expression of the $\mathrm{Na}(+) / \mathrm{H}(+)$ exchanger NHE3 in the kidney. Kidney Int 2005; 67: 1410-1419. 
30. Zuber AM, Centeno G, Pradervand S et al: Molecular clock is involved in predictive circadian adjustment of renal function. Proc Natl Acad Sci U S A 2009; 106: $16523-16528$.

31. Nikolaeva S, Pradervand S, Centeno $G$ et al: The circadian clock modulates renal sodium handling. J Am Soc Nephrol 2012; 23: 1019-1026.

32. Stow LR, Richards J, Cheng KY et al: The circadian protein period 1 contributes to blood pressure control and coordinately regulates renal sodium transport genes. Hypertension 2012; 59: 1151-1156.

33. Wu C, Sui G, Archer $\mathrm{S}$ et al: Expression of circadian clock gene Period 2 in the mammalian bladder identified by real-time imaging with luciferase reporter gene Presented at annual meeting of American Urological Association, Atlanta, Georgia, May 19-23, abstract: 259.

34. Cho Y S, Kim K H, Yoon S J et al: Possible involvement of bladder peripheral clock in the circadian control of voiding function. Presented at annual meeting of International Continence Society, Beijing, China, October 15-19, 2012, abstract: 425.

35. Tahara Y, Kuroda H, Saito K et al: In vivo monitoring of peripheral circadian clocks in the mouse. Curr Biol 2012; 22: 1029-1034.

36. Storch KF, Lipan O, Leykin I et al: Extensive and divergent circadian gene expression in liver and heart. Nature 2002; 417: 78-83.

37. Bray MS, Shaw CA, Moore MW et al: Disruption of the circadian clock within the cardiomyocyte influences myocardial contractile function, metabolism, and gene expression. Am J Physiol Heart Circ Physiol 2008; 294: H1036-H1047.

38. Boyle EI, Weng S, Gollub J et al: GO::TermFinder--open source software for accessing Gene Ontology information and finding significantly enriched Gene Ontology terms associated with a list of genes. Bioinformatics 2004; 20: 3710-3715.

39. Christ GJ, Day NS, Day M et al: Increased connexin43-mediated intercellular communication in a rat model of bladder overactivity in vivo. Am J Physiol Regul Integr Comp Physiol 2003; 284: R1241-1248.

40. Montaldo P, Tafuro L, Narciso V et al: Correlations between enuresis in children and nocturia in mothers. Scand J Urol Nephrol 2010; 44: 101-105.

41. Bower WF, Sit FK, Yeung CK: Nocturnal enuresis in adolescents and adults is associated with childhood elimination symptoms. J Urol 2006; 176: 1771-1775. 
42. Alstrup K, Graugaard-Jensen C, Rittig S et al: Abnormal diurnal rhythm of urine output following renal transplantation: the impact of blood pressure and diuretics. Transplant Proc 2010; 42: 3529-3536.

43. Mahler B, Kamperis K, Schroeder M et al: Sleep deprivation induces excess diuresis and natriuresis in healthy children. Am J Physiol Renal Physiol 2012; 302: F236-F243.

44. Kasukawa T, Sugimoto M, Hida A et al: Human blood metabolite timetable indicates internal body time. Proc Natl Acad Sci U S A 2012; 109: 15036-15041.

45. Froy O: Circadian rhythms, aging, and life span in mammals. Physiology (Bethesda) 2011; 26: 225-235.

46. Meredith AL, Wiler SW, Miller BH et al: BK calcium-activated potassium channels regulate circadian behavioral rhythms and pacemaker output. Nat Neurosci 2006; 9: 1041-1049.

47. Marijic J, Li Q, Song M et al: Decreased expression of voltage- and $\mathrm{Ca}(2+)$-activated $\mathrm{K}(+)$ channels in coronary smooth muscle during aging. Circ Res 2001; 88: 210-216.

48. Sugaya K, Nishijima S, Miyazato $M$ et al: Effects of melatonin and rilmazafone on nocturia in the elderly. J Int Med Res 2007; 35: 685-691.

49. Soda T, Masui K, Okuno $\mathrm{H}$ et al: Efficacy of nondrug lifestyle measures for the treatment of nocturia. J Urol 2010; 184: 1000-1004.

50. Yoshimura K, Nakayama T, Sekine A et al: B-type natriuretic peptide as an independent correlate of nocturnal voiding in Japanese women. Neurourol Urodyn 2012; 31: 1266-1271. 


\section{Legends}

Figure 1 Diurnal change in triad of micturition: arousal state in the brain, urine production rate in the kidneys and functional bladder capacity.

Figure 2 Diurnal change in micturition in humans, rats and mice. Humans from Nakamura $\mathrm{S}$ et al (ref 1) modified. N, number of patients. NS, not significant. $* P<0.05$, $* * P<0.01, * * * P<0.005$ and $* * * * P<0.001$. Mean \pm SD. Statistical significance between the 2 age groups was calculated with Student's $t$-test, and that between periods was calculated by paired $t$-test. Rats from Herrera GM et al (ref 20) modified. $P<0.05$ by 2 -tailed, unpaired $t$-test. Mice from Negoro $\mathrm{H}$ et al (ref 10) modified. WT mice $(n=$ 5), for 8 days under LD conditions and 5 days under DD conditions. Mean \pm SEM.

Figure 3 The circadian clock system. (A) Transcription-translation feedback mechanism in the core loop (upper row) and other loops of the circadian clock (lower row). Clock-controlled elements on the promoter are shown as the E/E' box, D box and RORE, and positive transcription factors (green ovals) and negative transcription factors (magenta ovals) are indicated. Some of clock genes have more than one clock controlled elements. Clock genes also regulate clock-controlled genes (CCGs). (B) The 
SCN tunes peripheral clocks via the hormonal system, autonomic nervous system and behavioral controls as feeding and sleep-awake cycles. SCN, suprachiasmatic nucleus. 

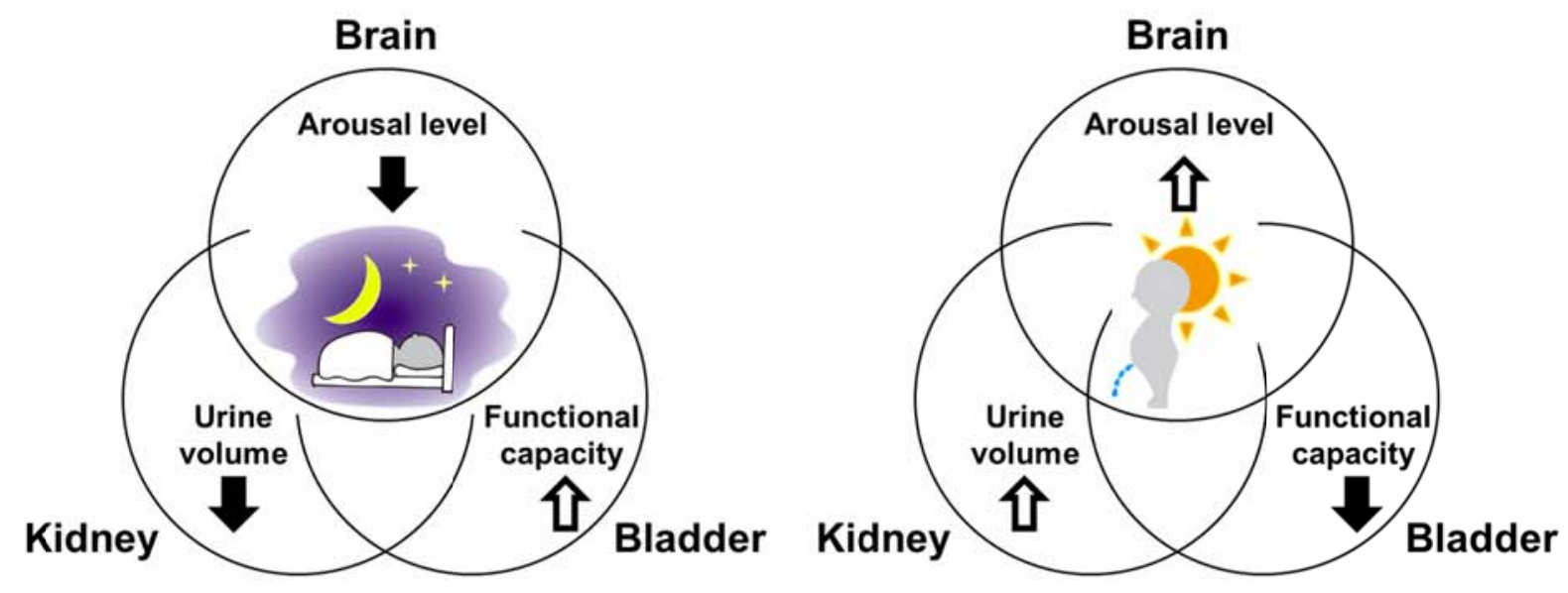

Figure 1 O.Ogawa et al

Bladder 
Figure 2 O.Ogawa et al

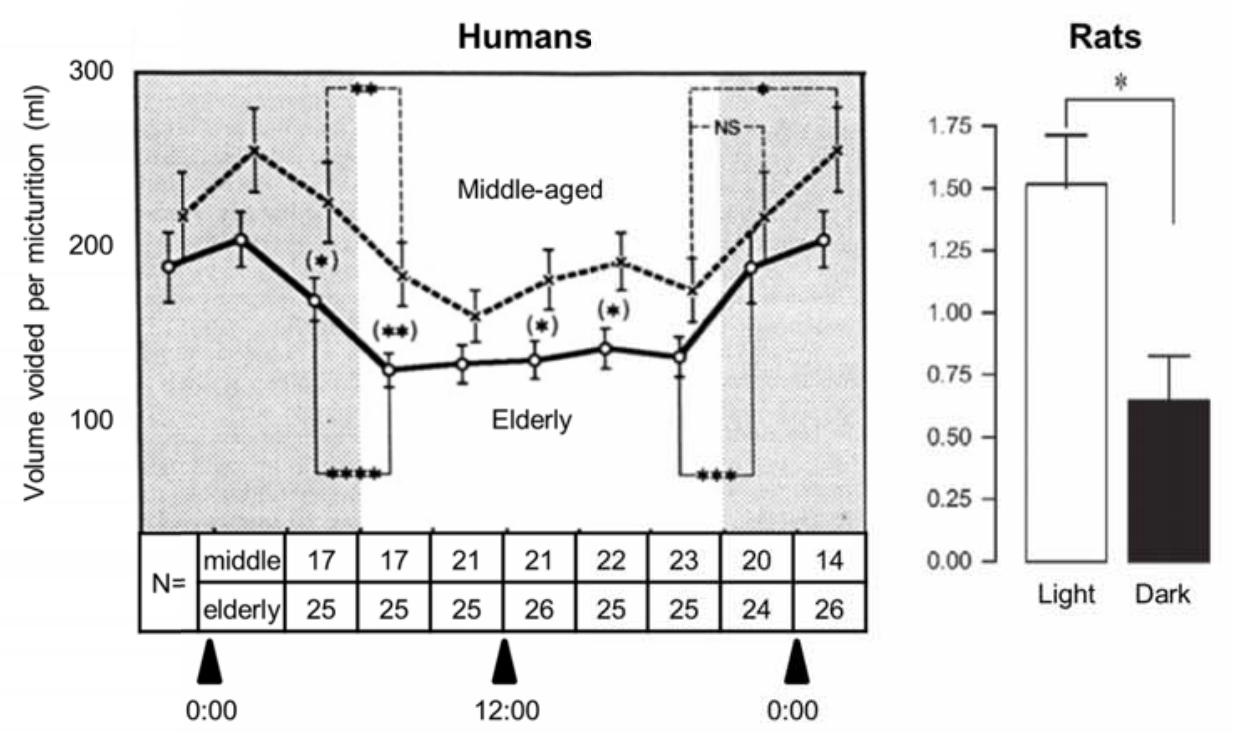

Mice

Light/Dark (LD) conditions, time (h)

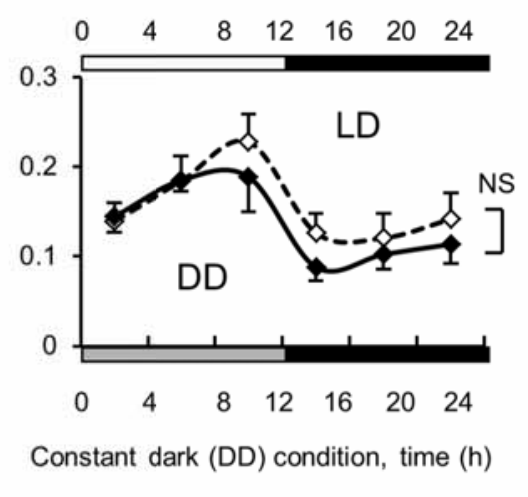


A
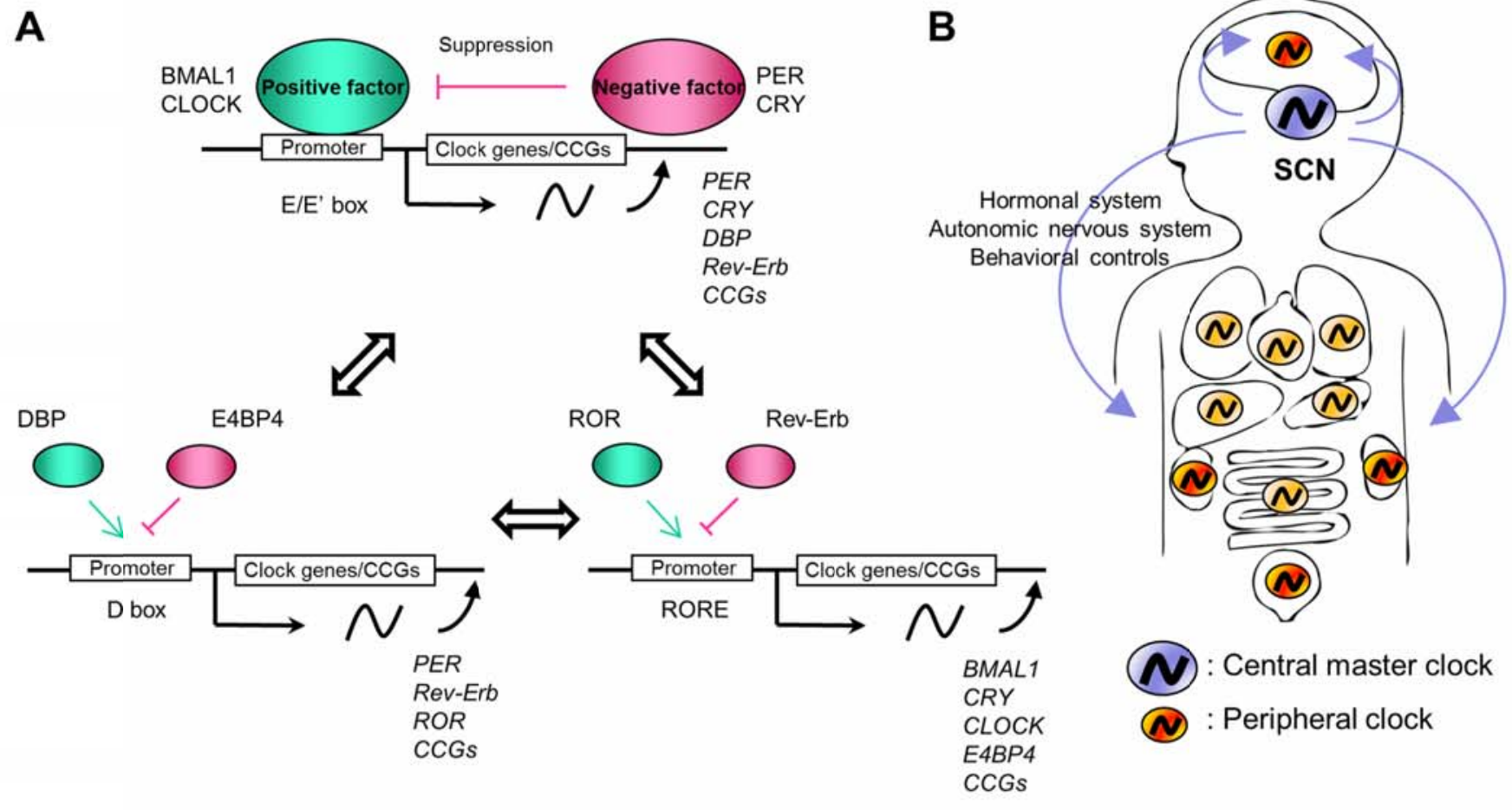

Figure 3 O.Ogawa et al 